\title{
Low-dose Glucocorticoid Use Does Not Reduce Biologic Use in Early RA, Nor Do Biologics Reduce the Need for Glucocorticoids
}

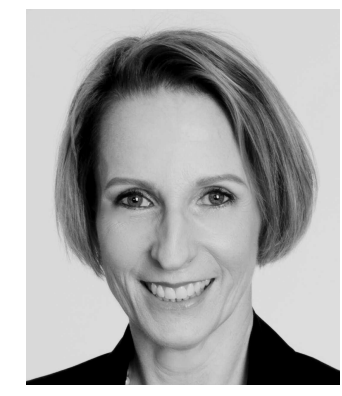

The persistent use of glucocorticoids (GC) has been associated with higher risks of osteoporosis, fractures, infection, and cardiovascular events. Although more costly, biologic disease-modifying antirheumatic drug (bDMARD) use could limit complications from prolonged exposures to $\mathrm{GC}$ in those patients at risk. The question arises as to whether this is occurring in settings of usual care. Patterns of bDMARD and GC use have rarely been studied in large populations, and factors that predict the initiation or persistent use of either or both are relevant to patients, providers, and payers. In this edition of The Journal, George, et al ${ }^{1}$ examined predictors associated with time to first bDMARD use from the time methotrexate (MTX) was first initiated (index date). GC users were those who had started GC within 90 days of the index date, and used GC consistently over the 2 -year study window. The study's 17,415-person rheumatoid arthritis (RA) cohort of incident MTX users, classified as RA using diagnostic codes, was treated between 2005 and 2016. Patients were identified from 3 linked US Veterans Affairs (VA) national administrative databases, and recorded data were used from real-world settings for 2 years from the index date. Initiation of bDMARD had to have been within 2 years of the index date. Inclusion criteria were intended to enrich for RA patients with active disease. Excluded patients were those whose RA may have been a mimic of polymyalgia rheumatica, or whose disease was easily controlled with GC or weaker DMARD, or those with other inflammatory forms of arthritis. Patients differed from those usually seen in practice in that only $12 \%$ were female, mean body mass index (BMI) was high (> 29), and there were notably high proportions of psychological and pulmonary comorbidities among current smokers (40\%).

The authors evaluated predictors of early bDMARD initiation and consistent GC use. Early initiation of bDMARD $(21 \%)$ was higher in younger patients $(<50 \mathrm{yrs})$, current smokers, seropositive patients, and those using $\mathrm{GC}^{1}$. Use of bDMARD increased with higher BMI. Early use of
bDMARD was lower in older adults, minorities, and users of other conventional synthetic (cs)DMARD. Presumably bDMARD use was avoided in those with specific comorbidities such as heart failure and cancer (as would be expected, given black box warnings against the use of many bDMARD when these conditions are present).

Interestingly, but not surprisingly, baseline (49\%) and persistent GC use (90\%) was high in this study ${ }^{1}$. Minorities, smokers, hydroxychloroquine users, and those with psychological and pulmonary comorbidities or high inflammatory markers were higher GC users, whereas women and those with diabetes, higher BMI, or prior csDMARD use had lower GC use. Persistent use of GC beyond 6-12 months of taking MTX showed a similar pattern. Importantly, persistent GC use remained high in those with depression, interstitial lung disease, seropositivity, a high C-reactive protein level, and current csDMARD use. This suggests that GC persistence may be associated with a poor prognosis phenotype that includes disease-related comorbid conditions, smoking, and high BMI. Moreover, the frequency of GC users did not decline with bDMARD use; however, it could not be determined from this database whether GC doses were actually reduced.

Despite some limitations in the generalizability of these data, observed associations are consistent with other early RA cohorts $^{2}$. Smoking rates were much higher and/or high BMI could also be driving incident comorbidities and persistent GC use, yet these comorbidities may limit eligibility for bDMARD. VA data do not provide GC dosage, thus it is unknown whether doses were reduced even though frequency of GC use was not reduced, even in those receiving bDMARD. If anything, bDMARD use was higher in GC users, suggesting use of GC did not limit the need for bDMARD. Males were predominant in their study, but 2000 females in the database were also studied, and the authors demonstrated that their findings were similar for both.

There may have been confounding by indication of

See Biologic initiation in RA, page 343

Personal non-commercial use only. The Journal of Rheumatology Copyright @ 2019 . All rights reserved. 
treatment in expected directions. There was less GC use in women, and in people with diabetes and high BMI, and more bDMARD use in younger, healthier people, without conditions associated with black box warnings. From these data, longstanding, poor prognosis RA requires more csDMARD, and risk-associated comorbidities result in more use of GC, contraindications for the use of bDMARD. This study population was enriched for specified conditions of concern, allowing the authors to demonstrate the issues at play for RA patients with a poor prognosis profile, aptly demonstrating potential treatment challenges.

Despite missing data on serology and absence of data on GC dosing, disease activity, and treatment response, this study demonstrates the treatment challenges in $\mathrm{RA}^{1}$. Whether the rate of bDMARD use in the cohort is high or low could be debated, but will remain unknown without determining how many patients reached treatment targets. However, GC use does not prevent what may be an even greater unidentified need for intensified treatment.

Thus, in this study of a very large population of patients with RA, GC use in itself may have been an indicator for severe RA, which if combined with concomitant lifestyle and RA-related comorbidities, resulted in persistence of GC use ${ }^{1}$. Key questions remain about optimizing the use of GC and bDMARD. Did bDMARD spare the amount of GC needed or vice versa? Were providers appropriately or overly cautious in trying to safely manage those contraindications to bDMARD, and did use of more GC in their place result in better outcomes? Would active interventions to reduce negative lifestyle factors in cohorts such as this improve outcomes and even lower needs for either bDMARD or GC? Finally, contrary to the notion that GC could be an alternative to bDMARD, GC may indicate the need for bDMARD, and will continue to be adjunctive therapy in most cases.

VIVIAN P. BYKERK (i), MD

Division of Rheumatology, Hospital for Special Surgery, Weill Cornell Medical College, New York, New York, USA.

Address correspondence to Dr. V.P. Bykerk, Hospital for Special Surgery, 535 East 70th St., New York, New York 10021, USA.

E-mail: bykerkv@hss.edu

\section{REFERENCES}

1. George MD, Sauer BC, Teng CC, Cannon GW, England BR, Kerr GS, et al. Biologic and glucocorticoid use after methotrexate initiation in patients with rheumatoid arthritis. J Rheumatol 2019;46:343-50

2. Barnabe C, Sun Y, Boire G, Hitchon CA, Haraoui B, Thorne JC, et al. Heterogeneous disease trajectories explain variable radiographic, function and quality of life outcomes in the Canadian Early Arthritis Cohort (CATCH). PLoS One 2015;10:e135327.

J Rheumatol 2019;46:331-2; doi:10.3899/jrheum.181262 